The Influence of Positive vs. Negative Affect on Multitasking

\author{
Brent Morgan ${ }^{1}$ and Sidney K. D’Mello \\ ${ }^{1}$ University of Memphis \\ Memphis, TN, USA 38152 \\ brent.morgan@memphis.edu \\ (corresponding author) \\ ${ }^{2}$ University of Notre Dame \\ Notre Dame, IN, USA \\ sdmello@nd.edu
}

Keywords: affect; emotion; mood; multitasking; task switching

Note: both authors have made equal contributions 


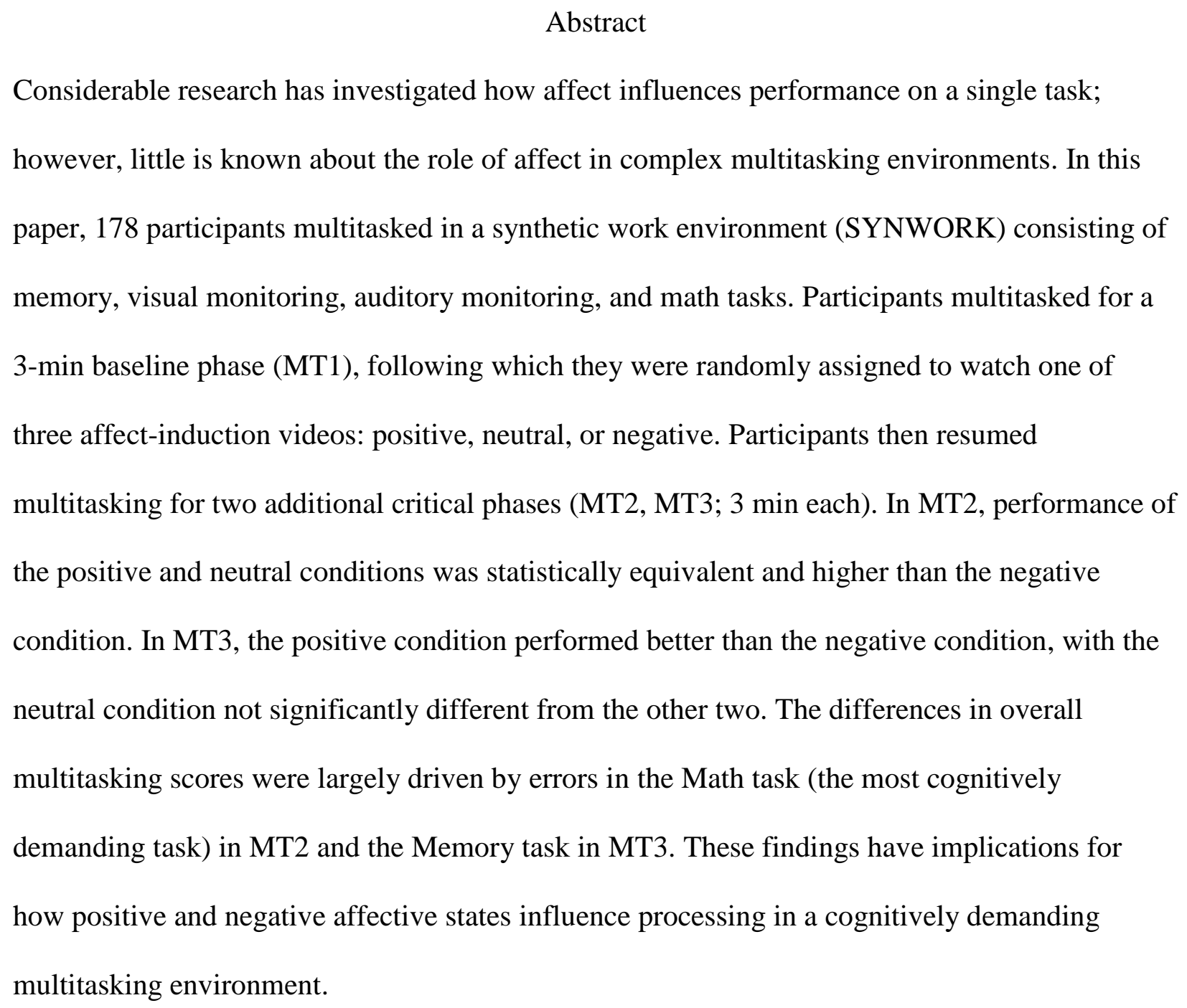




\section{The Influence of Positive vs. Negative Affect on Multitasking}

\section{Introduction}

Cognition and affect have historically been treated as separate entities (Storbeck \& Clore, 2007, Zajonc, 1980, 2000). However, researchers have come to appreciate the role that affect plays across numerous aspects of cognition, including working memory (Baddeley, 2013), cognitive flexibility (Van Wouwe, Band, \& Ridderinkhof, 2011), problem solving (Isen, Daubman, \& Nowicki, 1987), complex learning (D’Mello \& Graesser, 2012), and judgment and decision making (Blanchette \& Richards, 2010; Isen, 2000; Loewenstein \& Lerner, 2003; Schwarz, 2000). The vast majority of this research has focused on single task performance. Little is known about how affect influences multitasking, defined as performing two or more tasks simultaneously (dual-taking) or alternating over time spans of a few seconds (rapid task switching).

Previous findings pertaining to affect-performance relationships in single-task experiments may not generalize to multitasking environments because performing multiple tasks simultaneously is inherently different and can be more challenging than completing a single task (Monsell, 2003; Sauer, Wastell, \& Hockey, 1999). This is partly due to a 'switch cost' which occurs during rapid task switching (Monsell, 2003), which is the form of multitasking of interest in this study. The switch cost manifests as either additional time to complete the task, an increase in errors in task execution, or both. When multitasking, people can become overloaded as working memory and attentional resources become exhausted. Furthermore, overall performance can be adversely affected when the demands of one task interfere with those of another (Altmann \& Gray, 2008). Thus, with multitasking, the whole does not always equal the sum of the parts, so the role of affect during multitasking needs to be elucidated more systematically. In line with 
this, the purpose of this paper is to study the influence of positive and negative affect during multitasking.

\subsection{Affect and Cognition}

Researchers have investigated differences between positive and negative affect over a wide range of individual tasks, with the former considered to be superior in selective tasks (e.g., Fredrickson, 2003; Fredrickson \& Branigan, 2005; Lyubomirsky, King, \& Diener, 2005).

Negative affect, in contrast, has been shown to impede performance on numerous cognitive tasks, including executive functioning and memory (Marvel \& Paradiso, 2004). That being said, the liabilities of negative affect are hardly universal. Negative affect has been shown to be advantageous under particular conditions, such as performance appraisal (Sinclair, 1988), persuasion (Forgas, 2007), and complex problem-solving (Barth \& Funke, 2010; Fiedler, 1988). Chepenik, Cornew, and Farah (2007) also argue that the negative influence of negative affect on cognition is not near as pervasive as the literature suggests.

Cognitive processing mediates the influence of affect on task performance (Clore et al., 2001; Gasper, 2004; Gasper \& Clore, 2002). The levels of focus hypothesis (Gasper \& Clore, 2002) states that positive affect tends to promote global processing of information, whereas negative affect facilitates more local processing. Similar findings have shown that affect influences the scope of attention as well. (Fredrickson \& Branigan, 2005; Gasper \& Clore, 2002; Rowe, Hirsh, \& Anderson, 2007; Schmitz, De Rosa, \& Anderson, 2009; Uddenberg \& Shim, 2015). For example, the broaden-and-build theory (Fredrickson \& Branigan, 2005) posits that positive affect (i.e., happiness) begets a wider range of attention and thought-action repertoires than a neutral or negative affective state. Recent findings suggest that positive affect facilitates 
whichever focus is currently dominant (Huntsinger, Clore, \& Bar-Anan, 2010). That is, people in positive affective states have either a global or a local focus, depending on which of the two is primed. However, if neither is primed, people in a positive affective state would presumably adopt the global focus by default (Gasper \& Clore, 2002).

The global processing associated with positive affect can also influence performance on higher level tasks (such as set-switching) and cognitive flexibility (Braem et al., 2013; Dreisbach, 2006; Dreisbach \& Goschke, 2004; Dreisbach et al., 2005). In these situations, positive affect has a deleterious impact on performance when executing a to-be-maintained goal. For example, positive affect is associated with a wider field of vision (Schmitz, De Rosa, \& Anderson, 2009), which can result in greater distractibility (Rowe, Hirsh, \& Anderson, 2007). These distractions, in turn, can affect low-level perceptual tasks (e.g., flanker task; Rowe, Hirsh, \& Anderson, 2007). However, this broadening in visual attention may only occur for positively valenced stimuli (Wadlinger \& Isaacowitz, 2006). Thus, although people in a positive affective state are more flexible and open to environmental changes, this comes at the cost of increased distractibility (and reduced performance) when the task constraints remain constant.

Whereas positive affect increases visual attentional focus, negative affect (especially when combined with high arousal) narrows the scope of visual attention (Derryberry \& Tucker, 1994; Easterbrook, 1959). Similarly, whereas positive affect increases distractibility, negative affect improves selective attention (Finucane, 2011) and cognitive control (van Steenbergen, Band, \& Hommel, 2010). This allows for a deeper focus on the task at hand (Andrews \& Thomson, 2009; Braem et al., 2013). This is especially true for complex tasks, which can be decomposed into smaller, more manageable components where each component is addressed in turn (Andrews \& Thomson, 2009; Barth \& Funke, 2010). People in a negative affective state can 
solve complex problems because they can focus on the individual components without getting distracted by other components (Andrews \& Thomson, 2009; Braem et al., 2013). In sum, the research on the influence of affect during single task performance suggests that people in a positive affective state tend to see the forest, whereas people in a negative affective state focus on the trees.

\subsection{Affect and multitasking}

Although the vast majority of the research on affect has focused on single task performance, there has also been some studies on dual-task performance, which is one form of multitasking. For example, previous studies on affect in dual-task (visual + audio tasks) situations have focused on how affect reduces the attentional blink for the visual task (i.e., a reduction in the minimum refractory period before perceiving a second visual stimulus when dual-tasking). In particular, reductions in attentional blink have been associated with both positive affect (Olivers \& Nieuwenhuis, 2006) and sad mood (negative affect, low arousal; Jefferies, Smilek, Eich, \& Enns, 2008). Additionally, Rokke and colleagues found no changes in attentional blink associated with a mild dysphoric mood (Rokke, Arnell, Koch, \& Andrews, 2002).

It is important to point out that task switching is distinct from dual-tasking, though both are considered forms of multitasking (Posner, 1990). Rapid task switching requires switching from one task to another over short time spans, whereas divided-attention tasks require concurrent completion of individual tasks (Posner, 1990). Another important difference between rapid task switching and dual-tasking is the role of distraction. When a person engages in dualtasking, distractions may actually be beneficial because there is some evidence to suggest they 
can reduce the attentional blink (Arend, Johnston, \& Shapiro, 2006; Olivers \& Nieuwenhuis, 2005; 2006). When task switching, however, distraction shifts attention away from the task at hand, resulting in task switch costs (Monsell, 2003).

Overall, rapid task switching, which is the focus of this paper, is more complex and cognitively challenging than performing a single task, allowing for multiple approaches and strategies for task completion. For example, a person may view the environment as holistic (one large task) or as atomistic (multiple small tasks). If positive and negative affect facilitate opposing levels of focus (global vs. local), these differences should be clearly evident under the high cognitive demands of multitasking. Thus, the pertinent question is whether a more global, heuristics-driven approach engendered by positive affect or a more local, analytical approach associated with negative affect results in superior multitasking performance?

\subsection{Hypotheses and Current Study}

There are competing hypotheses on the influence of affect based on global vs. local cognitive processing. One hypothesis is that the global processing triggered by positive affect would improve multitasking performance because it would facilitate efficient processing strategies and the use of heuristics (Lucas \& Diener, 2003). Furthermore, an expansion of visual attention would improve a person's ability to monitor each individual task simultaneously. The alternate hypothesis is that additional task monitoring engendered by positive affect would be distracting (increasing task switching and therefore incurring more task switch costs), and heuristic-based processing would lead to more errors when focused attention on the environment is needed. Conversely, local processing engendered by negative affect would manifest as a more analytical and bottom-up approach. This more analytical approach would increase cognitive 
control, which is necessary for multitasking (Altmann \& Gray, 2008), and facilitate completion of individual tasks without interruption. That is, negative affect may be superior because the person would be more likely to focus on one task at a time (Andrews \& Thomson, 2009; Braem et al., 2013), thus avoiding the inherent performance costs associated with rapid task switching.

To test these hypotheses, we conducted an experiment in which participants multitasked during a baseline phase, watched a film designed to induce either a positive, neutral, or negative affective state, and then resumed multitasking for two experimental phases. We included separate experimental phases to measure both the immediate and delayed influence of affect on multitasking performance. Multitasking phases were kept short (3 minutes) for two reasons. First, high working memory demands (which would include multitasking) can "distract" a person from a negative affective state (Van Dillen \& Koole, 2007). Thus, it was imperative to have short multitasking phases to preserve group differences in affect. Second, multitasking can be exhausting, and we felt giving participants a break was appropriate so as to mitigate fatigue effects. Performance during the critical phases was compared across the three affect-induction conditions after covarying multitasking performance on previous phases.

We included a neutral condition to ascertain whether a difference between the positive and negative conditions was due to: a benefit to one affective state (state $1>[$ neutral = state 2]), a penalty to one affective state $([$ state $1=$ neutral $]>$ state 2$)$, or both $($ state $1>$ neutral $>$ state 2 ). Thus, the performance of the neutral condition should aid in explaining any differences between the positive and negative conditions (unless the neutral condition was either superior or inferior to both the positive and negative conditions). Considering that attending to global features (Navon, 1977) and information (Fiske \& Taylor, 1991) is the default processing strategy, the neutral condition would be expected to align more closely with the positive group. 


\section{Method}

\section{1 Participants}

The sample was composed of 178 participants recruited from Amazon Mechanical Turk (AMT). Recent research suggests that AMT is a reliable and valid source to collect experimental data and has confirmed that AMT samples are considerably more diverse than the typical undergraduate student population in the U.S. (Buhrmester, Kwang, \& Gosling, 2011; Mason \& Suri, 2012; Paolacci, Chandler, \& Ipeirotis, 2010; Rand, 2012). Participants were over 18, from the USA, and paid $\$ 1.25$ for their participation.

\subsection{Materials}

\subsubsection{SYNWORK (Multitasking Environment)}

SYNWORK (see Figure 1) is a computer-based multitasking environment composed of synthetic work tasks that are relevant to a number of occupations (e.g., naval ship operation; Elsmore, 1994). Environments such as SYNWORK are beneficial for such experiments because they afford both experimental control and realism (DiFonzo, Hantula, \& Bordia, 1998). SYNWORK requires participants to simultaneously attend to four individual tasks: Memory, Visual Monitoring, Auditory Monitoring, and Math (see Figure 1). Participants used a mouse to complete each task. Incorrect task completion resulted in an unpleasant sound, whereas there was no audible feedback for correct responses (as this tended to be distracting). The task was implemented as specified by Elsmore (1994), but with some modifications as noted below. It was programmed for online administration using Action Script 3 and can run on any browser that supports Adobe Flash. 


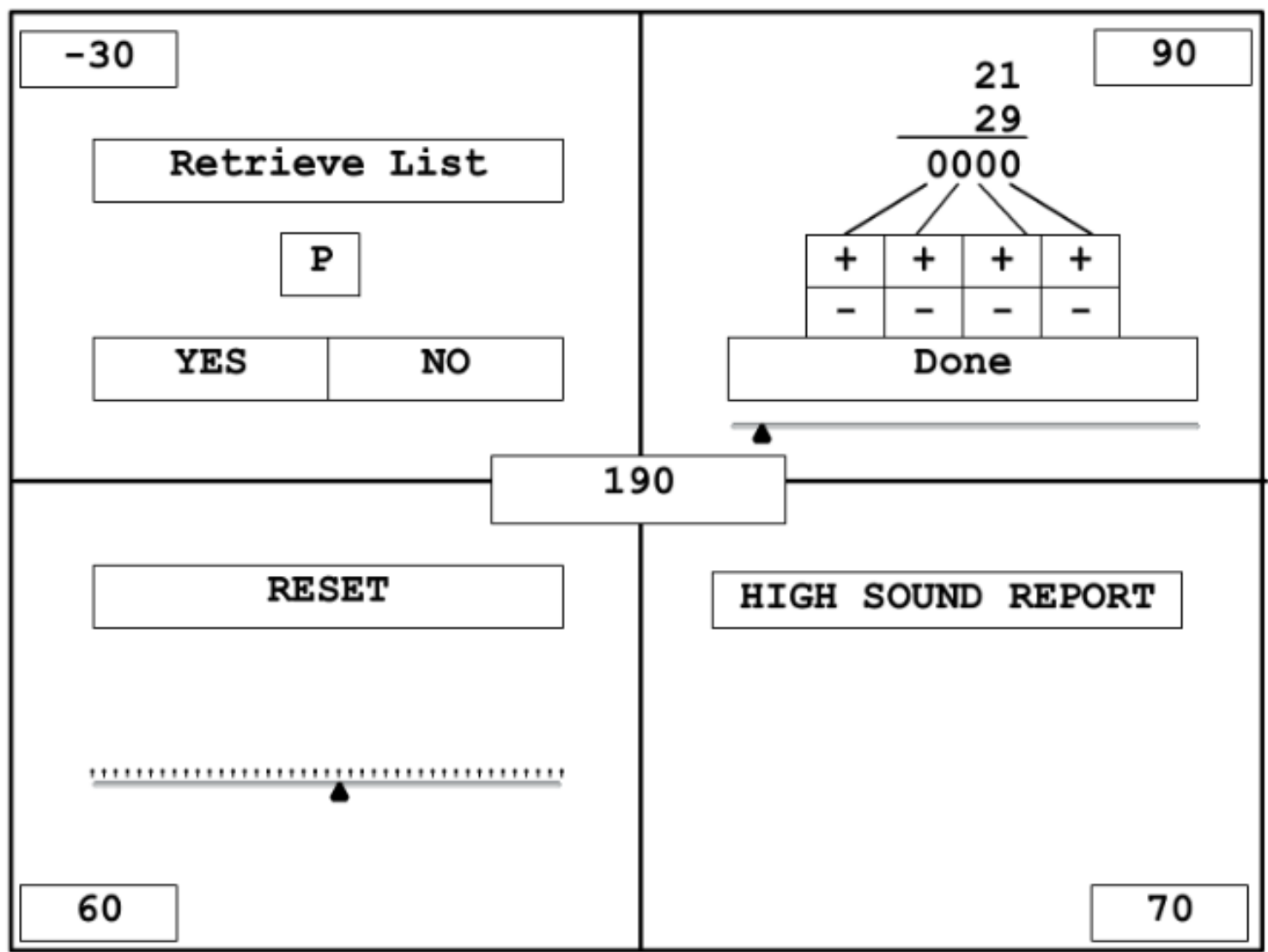

Figure 1. Screenshot of the SYNWORK Multitasking Environment. The four tasks, clockwise from top left: Memory, Math, Auditory Monitoring, Visual Monitoring.

\subsubsection{Memory}

In the top-left quadrant of the screen, participants were asked to remember a target list of six letters which were presented at the beginning of the task. The list was randomly chosen from a subset of the alphabet (minus C, D, M, Q, and V, which are often difficult to distinguish from other characters). After $5 \mathrm{~s}$, the target list disappeared, and a series of single-letter probes were displayed. Participants had to determine whether the probe letter was included in the target list. Participants could view the target list again by clicking "Retrieve List" at the cost of 10 points. Correct responses increased the participant's score by 10 points; incorrect responses decreased 
the score by 10 points. If the participant did not answer after $10 \mathrm{~s}, 10$ points were automatically deducted. After points were awarded/deducted, a new probe letter appeared after a ten second delay. The target list was refreshed at the start of each multitasking phase. Before each phase started, the instructions reminded participants to check the new target list.

\subsubsection{Visual Monitoring}

In the bottom-left quadrant, a one-dimensional horizontal scale was displayed with a triangular pointer which started at the center of the scale. The pointer always moved away from the center of the scale (either left or right) at the rate of 1 unit per second. The participants earned points by clicking a "Reset" button above the scale before the pointer reached the end of the scale. Participants received more points the closer the pointer was to the edge of the scale when the "Reset" button was clicked, up to a maximum of 10 . If the pointer reached the end of the scale however, 10 points were deducted at the same rate as the pointer's movement (i.e., 10 per s) until the "Reset" button was clicked. This results in a sudden rapid reduction in points at a rate of 10 points per second accompanied with continuous negative auditory feedback. Once the "Reset" button was clicked, the pointer returned to the center of the scale and the process repeated. Thus, successive clicking on the "Reset" button would not earn any points as the pointer would constantly be at zero.

\subsubsection{Auditory Monitoring}

In the bottom-right quadrant, participants were asked to respond to one of two auditory probes. If they heard a high-pitched sound (20\% of probes), they were to click the "High Sound Report” button, whereas they were to ignore a lower-pitched sound. Probes were presented at five second intervals. Participants received 10 points for a correct report, and lost 10 points for 
an incorrect report or a timeout (10s). Participants received training on how to discriminate between low vs. high pitch probes in a preliminary instruction phase.

\subsubsection{Math}

In the top-right quadrant, participants were asked to solve a two-digit arithmetic problem using the "+" and "--" boxes corresponding to each digit. For example, if the problem was $51+$ 42 (answer is 93), the participant would solve the problem by clicking the "+" button under the ones (rightmost) column three times, and clicking the "+" button under the tens column (second from right) nine times. Clicking the "+" box when there was a 9 did not reset the value to 0 , and likewise clicking the "-_ box when there was a 0 did not result in any change. Participants had 30s to solve each problem and clicked the "Done" button to submit their answer. A timer display below the "Done" button featured a pointer which moved from one end of the scale to the other as time decreased. This allowed participants to estimate how much time they had left to solve the problem. Correct solutions increased their score by 10; incorrect solutions resulted in a loss of 10 points; and failure to respond within 30s resulted in a loss of points at the rate of 10 points per second with continuous negative auditory feedback (similar to visual monitoring).

\subsubsection{Overall Score}

The overall score (displayed in the center of the screen) was the sum of the scores of the four SYNWORK tasks. Individual task scores were also displayed in the four corners of the screen, as shown in Figure 1. Scores could decrease rapidly during timeouts for the Visual Monitoring or Math tasks, thereby making it important to not ignore these tasks. The Math task was more cognitively challenging than the other three (Proctor, Wang, \& Pick, 1998). The Math task was also unique among the four in that it required multiple clicks to respond, and there was 
no latency following task completion; participants were immediately presented with a new problem to solve).

\subsubsection{Affect Induction Stimuli}

Participants watched film segments designed to elicit a positive, neutral, or negative affective state. The positive film ("Whose Line is it Anyway?"; $2 \mathrm{~m} 28 \mathrm{~s}$ ) featured an improv comedy routine. The neutral video comprised four clips ("Blue [1]”; "Blue [2]"; "Blue [3]”; “The Lover": $2 \mathrm{~m} 05 \mathrm{~s}$ total) of several dialogue-free scenes from two drama films. The negative film ("The Champ"; $2 \mathrm{~m} 45 \mathrm{~s}$ ) depicted a child witnessing the death of his father. These clips were selected because they have been extensively normed and used in several studies (Schaefer, Nils, Sanchez, \& Philippot, 2010).

\subsubsection{Affect Grid}

Participants' dimensional affect (valence and arousal) was measured using the Affect Grid (Figure 2; Russell, Weiss, \& Mendelsohn, 1989). The Affect Grid is a single-item affect measurement instrument consisting of a $9 \times 9$ (valence $\times$ arousal) grid, and is a validated measure of affect with adequate reliability (Cronbach's alpha $=.85)$, convergent validity (correlations of .90 or higher with similar scales of affect), and discriminant validity (correlations of .20 or less with dissimilar scales of affect). The arousal dimension ranges from low arousal (1) to high arousal (9), while the valence dimension ranges from unpleasant feelings (1) to pleasant feelings (9). The Affect Grid was presented on the computer screen and participants responded by using the mouse to select the cell anywhere on the grid that best represented their current affective state. The benefit of using the Affect Grid is that participants can report their affective 
state without the presence of affect labels, which can be interpreted differently across

individuals. It also affords collection of multiple affect responses as it is a single item measure.

\section{Please click on the box that represents how you feel at this moment (Click next when you are done)}

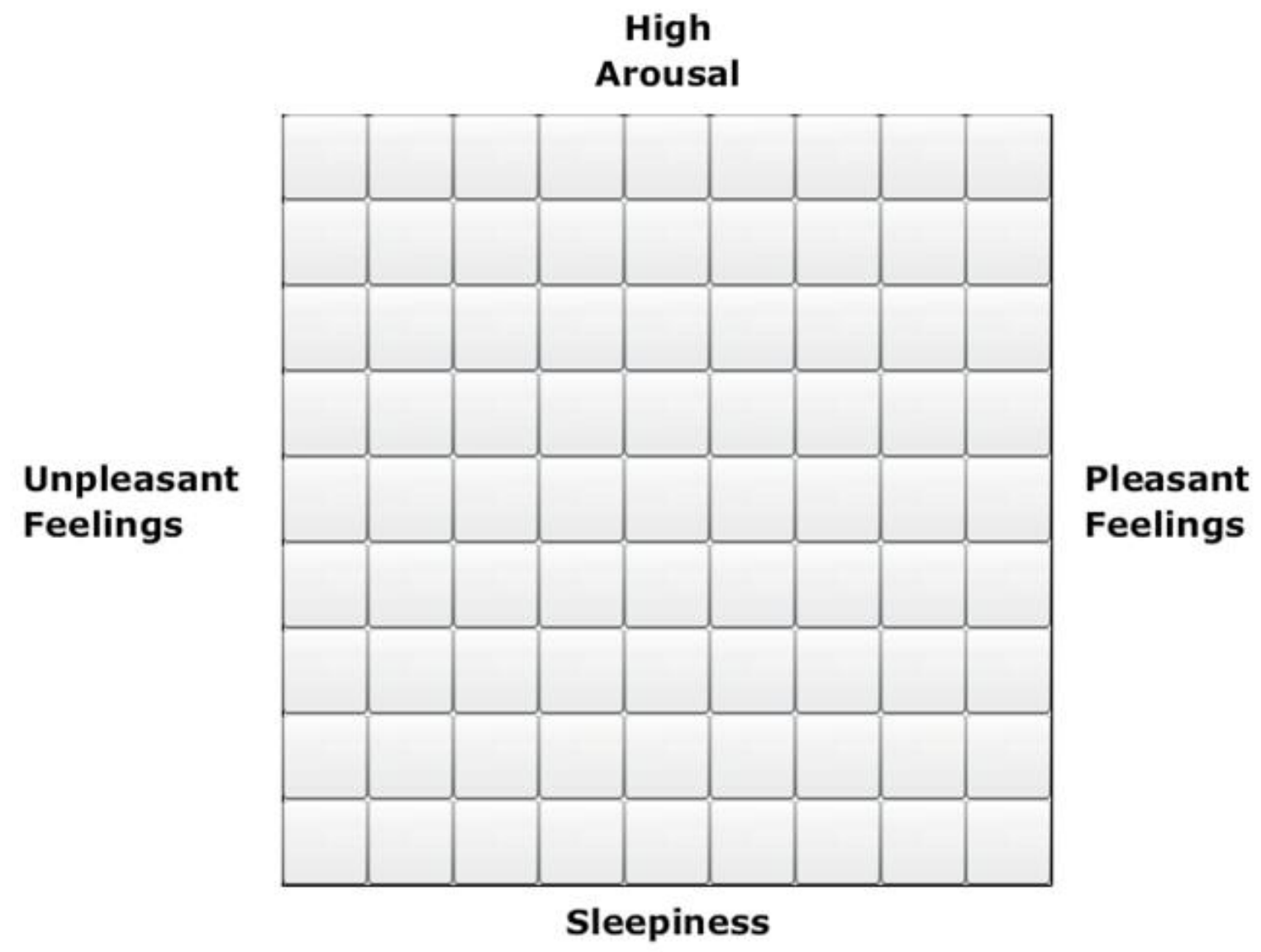

Figure 2. Screenshot of the Affect Grid, with valence on the vertical axis and arousal on the horizontal axis.

\subsection{Procedure}

Figure 3 depicts the various components of the protocol. Participants were first trained on how to use the Affect Grid using standard instructions from Russell, Weiss, \& Mendelsohn (1989), upon which they rated their affect. This was followed by training on individual SYNWORK tasks (45s each, except for math which was 60s) upon which the other tasks were 
hidden. After individual task training, all subsequent phases required the participants to switch between all four tasks (multitask) within the SYNWORK multitasking environment (see Figure 1). For all multitasking phases, participants were instructed to perform all four tasks together and reminded to check the new target list for the Memory task. Participants began by practicing multitasking for $1 \mathrm{~min}$. Pilot testing suggested that these were adequate training and practice time limits. After practicing all four tasks together, participants multitasked for 3 min at a baseline (Multitasking 1; MT1). Participants were then randomly assigned to one of three video conditions: positive ( $\mathrm{N}=47 ; 19$ females), neutral $(\mathrm{N}=66 ; 32$ females $)$, or negative $(\mathrm{N}=55 ; 25$ females). Following the video, participants self-reported their affective states with the Affect Grid (manipulation check). The multitasking score were then reset to zero, and they resumed multitasking for one experimental phase, Multitasking 2 (MT2). Participants self-reported their affect with the Affect Grid, then resumed multitasking for an additional experimental phase (MT3). The task parameters for MT2 and MT3 were identical to MT1 (3 min each). Finally, the negative condition was then asked to watch the positive-inducing film to ameliorate any negative effects attributed to the affect induction. Participants were then fully debriefed. 


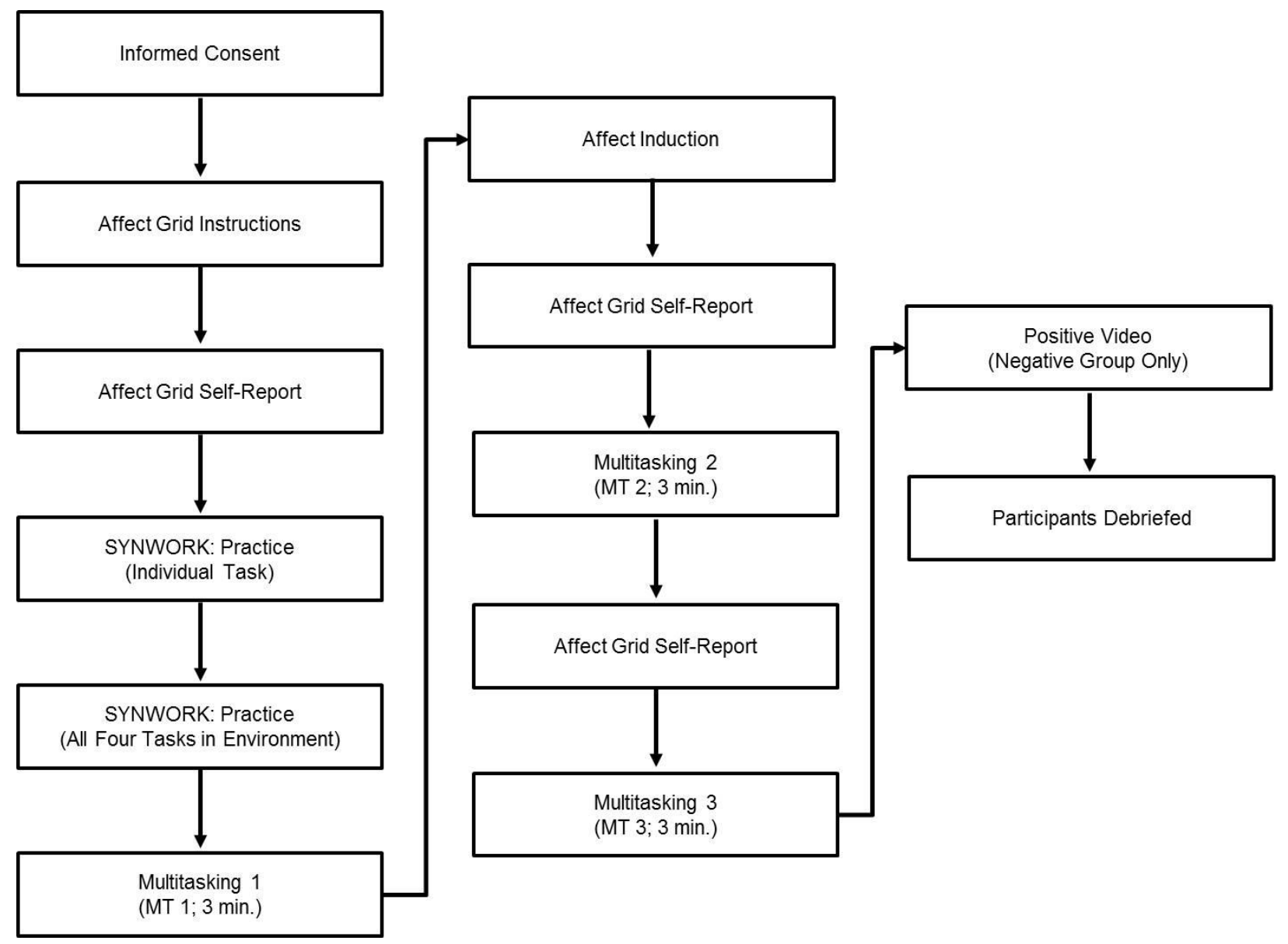

Figure 3. Experimental Protocol.

\section{Results}

We began by assessing the efficacy of the affect induction because a nontrivial percentage of people fail to respond to affect induction procedures (Martin, 1990). A total of 39 participants did not report an affect valence of 7-9 in the positive video condition $(N=7)$, 4-6 in the neutral video condition $(N=20)$, or $1-3$ in the negative video condition $(N=12)$. The $78 \%$ efficacy rate of our mood induction places it beyond the threshold of "highly effective" inductions (Gerrards-Hesse, Spies, \& Hesse, 1994).

Multitasking competency was also assessed in order to identify participants who did not fully attend to the instructions or did not meaningfully attempt the task. Ten participants had an 
overall score that was less than -500 on any one of the three multitasking phases. These ten participants were judged to have not adequately performed the task and were removed from the analyses. To mitigate outlier influence, since multitasking scores are theoretically unbounded, multitasking scores for the remaining 168 participants with z-scores $> \pm 3$ were recoded to the most extreme score within these bounds in their respective group.

\subsection{Affect Induction}

We began our affect analyses by ensuring there were no group differences before SYNWORK training. Table 1 presents these means and standard deviations for valence and arousal by video condition. Group differences in affect were assessed with one-way ANOVAs for each phase. The independent variable was video condition (positive, neutral, negative), with valence and arousal ratings as the dependent variables. Two one-way ANOVAs confirmed there were no differences for valence, $F(2,164)=2.03, p=0.14, \eta_{\mathrm{p}}^{2}=.024, M S E=2.21$, or arousal, $F(2,164)=0.284, p=0.75, \eta_{\mathrm{p}}^{2}=.003, M S E=2.71$ before the practice phase. We next assessed the efficacy of the affect induction procedure. After MT1, participants rated their mood twice: after watching the video (Post-Video), and after the experimental multitasking phase (PostMT2).

\section{Table 1}

Valence and Arousal Means (SD) by Condition

\begin{tabular}{lllll}
\hline & & \multicolumn{3}{c}{ Multitasking Phase } \\
& Condition & Pre-Practice & Post-Video & Post-MT2 \\
\hline \multirow{3}{*}{ Valence } & Positive & $6.02(1.42)$ & $7.55(1.44)$ & $5.91(1.65)$ \\
& Neutral & $6.05(1.60)$ & $5.11(1.54)$ & $5.74(1.83)$ \\
& Negative & $6.53(1.40)$ & $2.53(1.80)$ & $4.96(2.22)$ \\
Arousal & Positive & $5.26(1.65)$ & $6.66(1.36)$ & $7.32(1.45)$ \\
\hline
\end{tabular}




\begin{tabular}{llll}
\hline Neutral & $5.49(1.57)$ & $5.47(1.88)$ & $7.21(1.42)$ \\
Negative & $5.38(1.73)$ & $5.45(1.83)$ & $7.29(1.44)$ \\
\hline
\end{tabular}

MT $=$ Multitasking

For valence, there was a significant effect immediately after watching the video, $F(2$, $165)=125, p<.001, \eta_{\mathrm{p}}{ }^{2}=.602, M S E=2.580$. Comparisons revealed the following pattern in the data: positive $>$ neutral $>$ negative $(p<.001$ for all $)$. Thus, the videos were successful in inducing the intended affective state. There was also a significant effect for Post-MT2 valence ratings, $F(2,165)=3.74, p=.026, \eta_{\mathrm{p}}{ }^{2}=.043, M S E=3.69$. Comparisons revealed the following pattern $($ neutral $=$ positive,$p=.639)>$ negative, $p<.028$. Thus, while the positive and neutral groups were equivalent on valence after multitasking (MT2), the negative group still reported lower valence. In other words, positive affect wore off during the MT2 phase, but negative affect persisted.

For arousal, there was a significant effect after watching the video, $F(2,165)=8.06, p<$ $.001, \eta_{\mathrm{p}}{ }^{2}=.089, M S E=3.01$. Comparisons revealed the following pattern: (negative $=$ neutral, $p$ $=.962)<$ positive, $p<.001$. However, there were no differences among the groups at the PostMT2 phase, $F(2,165)=0.087, p=.917, \eta_{\mathrm{p}}{ }^{2}=.001, M S E=2.05$. Essentially, people in the positive video condition reported higher arousal immediately after the video, but otherwise there were no differences.

\subsection{Multitasking Performance}

We began by assessing whether there were group differences in SYNWORK scores (i.e., the sum of the four individual task scores) prior to the affect induction. Four one-way ANOVAs with respective SYNWORK task score as the dependent variable showed no single-task 
differences in the practice conditions for any of the four tasks, $F(2,165)<2.60, p>.077$.

Similarly, the groups were also equivalent in multitasking scores at MT1, $F(2,165)=0.124, p=$ 0.884; see Table 2 for descriptives).

Next, the influence of affective state on multitasking performance was assessed with two one-way ANCOVAs, one each for MT2 (immediate) and MT3 (delayed). The between-subjects independent variable was video condition (positive, neutral, negative), and the dependent variables were overall SYNWORK scores during phases MT2 and MT3. Baseline multitasking scores (MT1) were included as a covariate to control for individual differences in multitasking ability prior to the affect induction.

Table 2

Multitasking performance means (SD) by Condition and Phase

\begin{tabular}{lllll}
\hline \multirow{2}{*}{ Score } & Condition & MT1 & MT2 & MT3 \\
\hline \multirow{3}{*}{ Overall } & Positive & $267(135)$ & $336(141)$ & $388(96.7)$ \\
& Neutral & $256(185)$ & $327(127)$ & $348(136)$ \\
& Negative & $269(138)$ & $271(176)$ & $331(157)$ \\
& & & & \\
Memory & Positive & $62.1(85.9)$ & $89.2(86.4)$ & $121(47.4)$ \\
& Neutral & $62.0(91.4)$ & $96.7(71.3)$ & $99.2(71.1)$ \\
& Negative & $60.2(90.3)$ & $71.5(86.8)$ & $90.9(76.0)$ \\
Visual Monitoring & & & \\
& Positive & $74.0(25.0)$ & $71.8(26.4)$ & $75.6(21.3)$ \\
& Neutral & $64.0(42.9)$ & $68.2(32.5)$ & $68.9(30.3)$ \\
& Negative & $67.8(32.5)$ & $69.7(32.3)$ & $65.1(35.0)$ \\
Audio Monitoring & & & & \\
& Positive & $42.6(33.7)$ & $48.1(37.3)$ & $54.5(23.3)$ \\
& Neutral & $38.0(29.2)$ & $45.6(26.3)$ & $47.1(29.5)$ \\
& Negative & $44.2(36.8)$ & $37.3(43.0)$ & $47.1(36.0)$ \\
& & & & \\
Math & Positive & $93.2(77.7)$ & $132(40.4)$ & $139(54.6)$ \\
& Neutral & $98.0(73.4)$ & $116(64.2)$ & $131(71.9)$ \\
& Negative & $93.3(71.4)$ & $95.1(81.8)$ & $131(59.2)$ \\
\hline
\end{tabular}

MT $=$ Multitasking 
The results for MT2 revealed a significant main effect for video condition, $F(2,164)=$ $5.26, p=.006, \eta_{\mathrm{p}}{ }^{2}=.060, M S E=14,700$. Comparisons revealed the following pattern in the data: $($ positive $=$ neutral, $p=.887)>$ negative, $p<.007$ for both. Given the difference in PostVideo arousal scores, a separate model was tested which included Post-Video arousal score as a covariate; however, it was not a statistically significant covariate $(p=.892)$, and it had no influence on the main effect. Hence, it was removed from the final model.

The results for MT3 also revealed a significant main effect for video condition, $F(2,164)$ $=3.77, p=.025, \eta_{\mathrm{p}}^{2}=.044, M S E=11,300$. Comparisons revealed the following pattern in the data: positive $>$ negative, $p=.007)$. There were no differences between the neutral group and either positive or negative, $p>.093$ for both.

Thus, the positive video condition outperformed the negative video condition in both experimental multitasking phases. At MT2, the neutral group was equal to the positive group and superior to the negative group. At MT3, however, the mean for the neutral group was between the positive and negative groups, but not significantly different from either. The covariateadjusted means for each condition and phase are displayed in Figure 4. 


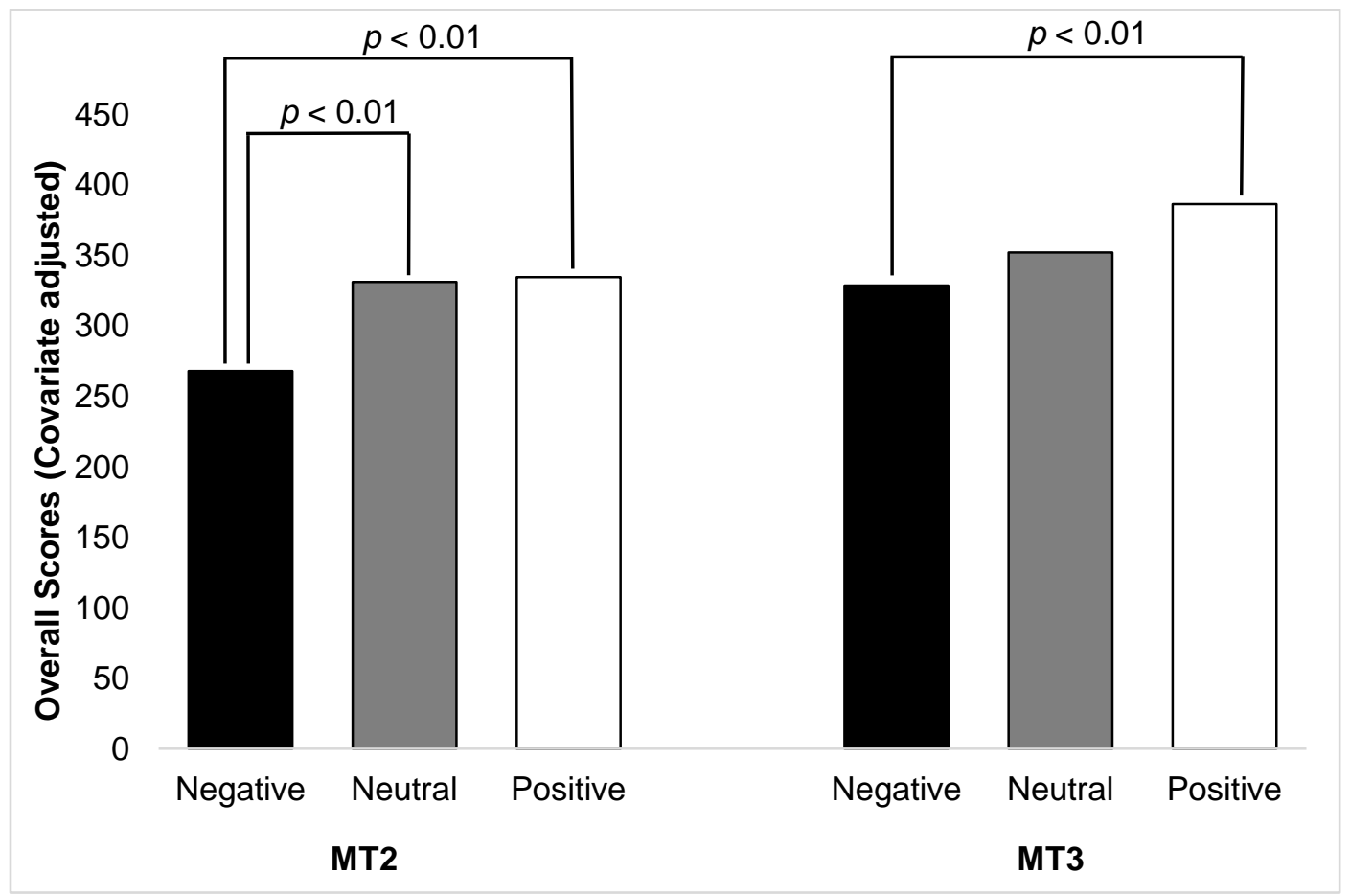

Figure 4. Overall SYNWORK scores by condition and phase (adjusted for Baseline performance).

\subsubsection{Individual Tasks}

After comparing overall multitasking scores, the next question was whether these differences could be attributed by large differences in one or more individual tasks, or a combination of smaller differences across all four tasks. Accordingly, the four individual SYNWORK tasks were analyzed using separate ANCOVAs (these and remaining analyses use the identical format as the previous analyses on overall scores). For MT2, there were no differences between video conditions for the Visual Monitoring, $F(2,164)=0.132, p=.876, \eta_{\mathrm{p}}{ }^{2}$ $=.002, M S E=524$, Auditory Monitoring, $F(2,164)=2.23, p=.111, \eta_{\mathrm{p}}{ }^{2}=.026, M S E=1050$, or 
Memory, $F(2,164)=1.93, p=.149, \eta_{\mathrm{p}}{ }^{2}=.023, M S E=4720$, tasks. However, there was a significant difference for the Math task, $F(2,164)=5.91, p=.003, \eta_{\mathrm{p}}{ }^{2}=.067, M S E=2920$. Comparisons revealed the following pattern: positive $>$ negative, $p=.001$ (see Table 2 for descriptive statistics). There was no difference between the neutral group and either positive or negative, $p>.061$ for both. Thus, the differences in multitasking scores between the positive and negative conditions at MT2 could largely be attributed to lower performance on the Math task for the negative group.

For MT3, there were no significant differences between video conditions for the Visual Monitoring, $F(2,164)=1.36, p=.261, \eta_{\mathrm{p}}^{2}=.016, M S E=587$, Auditory Monitoring, $F(2,164)=$ $0.973, p=.380, \eta_{\mathrm{p}}{ }^{2}=.012, M S E=880$, or Math, $F(2,164)=0.533, p=.588, \eta_{\mathrm{p}}{ }^{2}=.006, M S E=$ 2740, tasks. However, there was a significant difference for the Memory task, $F(2,164)=3.08, p$ $=.049, \eta_{\mathrm{p}}^{2}=.036, M S E=3,750$. Comparisons revealed the following pattern: positive $>$ negative, $p=.017$. There were no differences between the neutral group and either positive or negative, $p>.067$ for both. Thus, the differences in multitasking scores between the positive and negative conditions at MT3 could largely be attributed to higher performance on the Memory task for the positive group.

\subsubsection{Error Analysis}

We next assessed whether the differences in the two individual tasks were caused by participant errors in completing each task (i.e., the Math task in MT2 and the Memory task in MT3). Error rates were computed as incorrect task attempts (but not timeouts) divided by total task attempts (see Table 3 for descriptives). That is, an error was considered to have occurred when a participant attempted a task and produced an incorrect response. Failures to attempt the task (i.e., task timeouts) were not included as they were both rare and difficult to interpret. For 
example, a participant may not complete a task for a number of reasons: inability to complete the task on time, forgetting to attend to the task, or even electing to ignore the task as a strategy.

Thus, the focus is on errors of commission rather than errors of omission.

\section{Table 3}

Multitasking mean error rates (SD) by Condition and Phase

\begin{tabular}{|c|c|c|c|c|}
\hline \multirow[b]{2}{*}{ Task } & \multirow[b]{2}{*}{ Condition } & \multicolumn{3}{|c|}{ Multitasking Phase } \\
\hline & & MT1 & MT2 & MT3 \\
\hline \multirow{3}{*}{ Memory Error Rates } & Positive & $0.32(0.26)$ & $0.24(0.25)$ & $0.15(0.14)$ \\
\hline & Neutral & $0.32(0.26)$ & $0.23(0.21)$ & $0.21(0.21)$ \\
\hline & Negative & $0.33(0.27)$ & $0.28(0.24)$ & $0.23(0.22)$ \\
\hline \multirow{3}{*}{ Math Error Rates } & Positive & $0.11(0.11)$ & $0.08(0.08)$ & $0.08(0.08)$ \\
\hline & Neutral & $0.12(0.13)$ & $0.10(0.10)$ & $0.10(0.10)$ \\
\hline & Negative & $0.10(0.10)$ & $0.13(0.11)$ & $0.08(0.09)$ \\
\hline \multirow{3}{*}{ Math Click Error Rates } & Positive & $0.05(0.04)$ & $0.03(0.02)$ & $0.04(0.03)$ \\
\hline & Neutral & $0.05(0.05)$ & $0.04(0.03)$ & $0.03(0.03)$ \\
\hline & Negative & $0.05(0.04)$ & $0.05(0.03)$ & $0.04(0.03)$ \\
\hline
\end{tabular}

For MT2, there was a significant finding for error rates in the Math task, $F(2,164)=$ 3.93, $p=.022, \eta_{\mathrm{p}}{ }^{2}=.046, M S E=.009$. Comparisons revealed the following pattern: (positive $=$ neutral, $p=.532)<$ negative, $p<.031$ for both, which inverts the pattern with MT2 overall scores and MT2 math scores (i.e., more errors so lower scores). Given the difference in MT2 Math error rates, we also analyzed clicking errors in the Math task, specifically the proportion of times participants clicked the "_," box (relative to clicking the "+" box). As mentioned in the Methods section, clicking the "_-" box for a 0 would not "reduce" the 0 to a 9 . Hence, any click of the "_-" button offered no strategic advantage and would thus likely be the result of too many clicks of the "+" button. Use of the "__" button could arise from a miscalculation or 
overaggressive clicking, but represents an error in either case. The results were significant, $F(2$, $164)=6.03, p=.003, \eta_{\mathrm{p}}{ }^{2}=.068, M S E=0.001$. Comparisons revealed the following pattern: positive $(p<.048$ for both $)<($ negative $=$ neutral, $p=.092)$, indicating a lower proportion of " " box click errors for the positive group.

For MT3, there was a significant finding for error rates in the Memory task, $F(2,164)=$ $3.13, p=.047, \eta_{\mathrm{p}}^{2}=.037, M S E=.033$. Comparisons revealed the following pattern: positive < negative, $p=.016$. There were no differences between the neutral group and either positive or negative, $p>.065$ for both. This replicates the pattern with overall MT3 scores and MT3 Memory scores (in the inverse). Given the difference in MT3 Memory error rates, we also analyzed judgment errors in the Memory task, specifically whether errors arose from incorrectly clicking "Yes" vs. incorrectly clicking "No". The results were not significant, $F(2,164)=0.547$, $p=.580, \eta_{\mathrm{p}}^{2}=.007, M S E=3.070$, indicating that both error types were equally likely.

\subsubsection{Clicks and Transitions}

Lastly, there was the question of whether affective state affected quantity rather than quality of performance. For example, it is possible that participants' affective state merely affected the volume of clicking rather than accuracy. However, the results indicated no difference in the number of clicks among the video conditions, $F(2,164)=0.073, p=.930, \eta_{\mathrm{p}}{ }^{2}=$ $.001, M S E=1,520$ for MT2, and $F(2,164)=0.662, p=.517, \eta_{\mathrm{p}}{ }^{2}=.008, M S E=1,950$ for MT3. Similarly, there were no differences in the number of overall transitions from one task to another, $F(2,164)=2.14, p=.121, \eta_{\mathrm{p}}{ }^{2}=.025, M S E=1480$ for MT2, and $F(2,164)=0.336, p=.715, \eta_{\mathrm{p}}{ }^{2}$ $=.004, M S E=1760$ for MT3. Thus, the performance difference of one condition over another was not due to clicking more, but rather clicking more accurately.

\section{Discussion}


The influence of affect on multitasking performance was assessed by having participants multitask before and after watching a positive, neutral, or negative video. Multiple posttest phases were included to assess the immediate and slightly delayed influence of affect. Analyses of the immediate influence of affect on multitasking performance revealed a penalty for negative affect (i.e., the negative group had lower performance relative to the other two groups). In the delayed experimental phase, the positive group again outperformed the negative group, with neutral not different from either. Thus, the positive group was superior to the negative group in both phases. It appears that negative affect may increase task switch costs (Andrews \& Thomson, 2009), thereby hindering multitasking performance in the immediate experimental phase.

Group differences in overall multitasking performance were reflected in individual task performance. The positive group outperformed the negative group in the Math task in the immediate experimental phase (directly following the affect induction). The Math task is more cognitively challenging than the other three SYNWORK tasks (Proctor, Wang, \& Pick, 1998), and task switch costs increase with task complexity (Rubinstein, Meyer, \& Evans, 2001). Thus, group differences in task switch costs may be more pronounced in more difficult tasks. In the delayed phase, the positive group again outperformed the negative group, but on the Memory task. This finding might be explained by the fact that negative affect has been associated with lower performance on working memory tasks (specifically N-back tasks; Brose, Schmiedek, Lövdén, \& Lindenberger, 2012).

Error analyses of the two tasks with group differences (Math, Memory) revealed patterns that were consistent with overall and individual task performance. In the immediate phase, the poor performance of the negative group in the Math task could be attributed to a higher error rate when solving the problem. The negative group was also associated with a higher proportion of 
low-level click errors than the positive group. In the delayed phase, the negative group exhibited a higher rate of errors on the Memory task than the positive group. Other studies have reported that negative affect is also associated with reduced error awareness and saliency (Hajcak, McDonald, \& Simons, 2004; Luu, Collins, \& Tucker, 2000).

To what extent do the data support our hypotheses? The first hypothesis stated that global processing would improve multitasking performance via efficient cognitive functioning and broadened visual attention. Although the positive affect group had higher multitasking performance than negative group for both the immediate and delayed phases, it did not outperform the neutral condition on either phase, suggesting no benefit to positive affect (compared to neutral) when multitasking. Previous studies have indicated that positive affect facilitates executive functioning, including cognitive flexibility (Van Wouwe, Band, \& Ridderinkhof, 2011), problem-solving (Isen, Daubman, \& Nowicki, 1987), decision making (Isen \& Means, 1983; Isen, 2000), and working memory (Yang \& Yang, 2014). Moreover, this facilitation improves performance on tasks with high cognitive demand (Isen, Daubman, \& Nowicki, 1987), which would include multitasking (Monsell, 2003; Sauer, Wastell, \& Hockey, 1999). However, the positive affect group showed no statistically significant improvement over the neutral affect group, so there is no evidence to support positive facilitation hypothesis.

According to the second hypothesis, local, effortful processing by the negative group would facilitate cognitive control and selective attention, improving multitasking performance (Finucane, 2011; van Steenbergen, Band, \& Hommel, 2010). However, the increase in avoidable errors in the Math task - a relatively simple arithmetic task - suggests that this was not the case. In fact, negative affect has been associated with lapses in attentional control (Smallwood, Fitzgerald, Miles, \& Phillips, 2009), and may even be exacerbated under acute stress (Vinski \& 
Watter, 2013), which can occur during multitasking (Wetherell \& Sidgreaves, 2005). Thus, there was no evidence in support of the second hypothesis as well. In fact, the data support a temporary negative hindrance hypothesis since the negative group had lower performance than both the positive and neutral groups during the immediate multitasking phase.

Like most studies, some limitations of this study need be addressed in future work. For one, participants multitasked over a relatively short time span, so the medium- to long-term influence of affect on multitasking performance is unknown. Second, affective influences may be moderated by relevant individual differences (e.g., working memory; Curci, Lanciano, Soleti, \& Rimé, 2013) which were not assessed in this experiment. Third, although SYNWORK is a domain-general multitasking environment, it remains to be seen if these findings will generalize to other multitasking environments, both domain-general and domain-specific. Lastly, this paper was concerned with affective valence rather than discrete affective states (Lerner, \& Keltner, 2000); it is likely that not all negative or positive affective states influence performance in the same way (Jefferies et al., 2008).

Future research could also provide a more thorough examination of multitasking behaviors exhibited under various different affective states. For example, eye tracking can provide additional insight into task switching behaviors (Mayr, Kuhns, \& Rieter, 2013) and possibly reveal more subtle differences between affective states. Furthermore, there is the question of whether affective states affect not only multitasking ability, but multitasking adaptability as well, as these may be different constructs (Morgan et al., 2013).

\section{Conclusions}

Although the idea that individuals in a positive affective state might be more effective multitaskers than negative ones is not new (e.g., Lucas \& Diener, 2003), the present experiment 
provides causal evidence with data collected in a simulated work environment. It also shows that the effect was not due to a performance boost attributable to positive affect, but rather to a temporary performance detriment caused by negative affect. These experimental findings complement recent correlational findings that paint a grim picture of negative affect and multitasking: not only is negative affect associated with greater multitasking frequency (Becker, Alzahabi, \& Hopwood, 2013) and duration (Calderwood, Ackerman, \& Conklin, 2014), but individuals in a negative affective state may have a harder time regulating their emotions (Pea et al., 2012).

The current findings have important implications for practitioners and researchers in human affect and performance. First, affect does appear to have an influence on multitasking performance, in line with numerous studies in single-task environments. Second, negative affect had a deleterious influence on multitasking performance, caused in part by an increase in clicklevel and task-level errors. Lastly, positive affect did not increase multitasking performance relative to neutral affect, despite numerous studies showing positive affect facilitating executive functioning across a number of individual tasks (Braem et al., 2013; Dreisbach, 2006; Dreisbach \& Goschke, 2004; Dreisbach et al., 2005; Isen, Daubman, \& Nowicki, 1987; Isen \& Means, 1983; Van Wouwe, Band, \& Ridderinkhof, 2011; Yang \& Yang, 2014). Hence, current understandings of affect in single tasks may not translate to multitasking environments that are increasingly ubiquitous in the $21^{\text {st }}$ century.

\section{Acknowledgement}

This research was supported by Sandia National Labs and the National Science Foundation (NSF) (HCC 0834847, and DRL 1235958). Any opinions, findings and conclusions, 
or recommendations expressed in this paper are those of the authors and do not necessarily reflect the views of the funding agencies. 


\section{References}

Altmann, E. M., \& Gray, W. D. (2008). An integrated model of cognitive control in task switching. Psychological Review, 115, 602-639.

Andrews, P. W., \& Thomson Jr, J. A. (2009). The bright side of being blue: depression as an adaptation for analyzing complex problems. Psychological Review, 116(3), 620-654.

Arend, I., Johnston, S., \& Shapiro, K. (2006). Task-irrelevant visual motion and flicker attenuate the attentional blink. Psychonomic Bulletin \& Review, 13(4), 600-607.

Baddeley, A. (2013). Working memory and emotion: ruminations on a theory of depression. Review of General Psychology, doi: 10.1037/a0030029.

Barth, C. M., \& Funke, J. (2010). Negative affective environments improve complex solving performance. Cognition and Emotion, 24(7), 1259-1268.

Baumann, N., \& Kuhl, J. (2005). Positive affect and flexibility: Overcoming the precedence of global over local processing of visual information. Motivation and Emotion, 29(2), 123134.

Becker, M. W., Alzahabi, R., \& Hopwood, C. J. (2013). Media Multitasking Is Associated with symptoms of depression and social anxiety. Cyberpsychology, Behavior, and Social Networking, 16(2), 132-135.

Blanchette, I., \& Richards, A. (2010). The influence of affect on higher level cognition: A review of research on interpretation, judgement, decision making and reasoning. Cognition \& Emotion, 24(4), 561-595.

Braem, S., King, J. A., Korb, F. M., Krebs, R. M., Notebaert, W., \& Egner, T. (2013). Affective modulation of cognitive control is determined by performance-contingency and mediated by ventromedial prefrontal and cingulate cortex. The Journal of Neuroscience, 33(43), $16961-16970$. 
Brose, A., Schmiedek, F., Lövdén, M., \& Lindenberger, U. (2012). Daily variability in working memory is coupled with negative affect: the role of attention and motivation. Emotion, 12(3), 605-617.

Buhrmester, M., Kwang, T., \& Gosling, S. D. (2011). Amazon's Mechanical Turk a new source of inexpensive, yet high-quality, data? Perspectives on Psychological Science, 6(1), 3-5.

Calderwood, C., Ackerman, P. L., \& Conklin, E. M. (2014). What else do college students "do" while studying? An investigation of multitasking. Computers \& Education, 75, 19-29.

Chepenik, L. G., Cornew, L. A., \& Farah, M. J. (2007). The influence of sad mood on cognition. Emotion, 7(4), 802-811.

Clore, G.L., Wyer, R.S., Dienes, B., Gasper, K., Gohm, C., \& Isbell, L. (2001). Affective feelings as feedback: Some cognitive consequences. In L.L. Martin \& G.L. Clore (Eds.), Theories of mood and cognition: A user's guide (pp. 27-62). Mahwah, NJ: Erlbaum.

Curci, A., Lanciano, T., Soleti, E., \& Rimé, B. (2013). Negative emotional experiences arouse rumination and affect working memory capacity. Emotion,13(5), 867-880.

D’Mello, S. K., \& Graesser, A. C. (2012). Emotions during learning with AutoTutor. In P. Durlach \& A. Lesgold (Eds.), Adaptive technologies for training and education. Cambridge, UK: Cambridge University Press.

Derryberry, D., \& Tucker, D. M. (1994). Motivating the focus of attention. In P. M. Niedenthal and S. Kitayama (Eds.), The heart's eye: Emotional influences in perception and attention. London: Academic Press.

DiFonzo, N., Hantula, D. A., \& Bordia, P. (1998). Microworlds for experimental research: Having your (control and collection) cake, and realism too. Behavior Research Methods, 30(2), 278-286. 
Dreisbach, G. (2006). How positive affect modulates cognitive control: The costs and benefits of reduced maintenance capability. Brain and Cognition, 60(1), 11-19.

Dreisbach, G., \& Goschke, T. (2004). How positive affect modulates cognitive control: reduced perseveration at the cost of increased distractibility. Journal of Experimental Psychology: Learning, Memory, and Cognition, 30(2), 343-353.

Dreisbach, G., Müller, J., Goschke, T., Strobel, A., Schulze, K., Lesch, K. P., \& Brocke, B. (2005). Dopamine and cognitive control: the influence of spontaneous eyeblink rate and dopamine gene polymorphisms on perseveration and distractibility. Behavioral Neuroscience, 119(2), 483-490.

Easterbrook, J. A. (1959). The effect of emotion on cue utilization and the organization of behavior. Psychological Review, 66(3), 183-201.

Elsmore, T. F. (1994). SYNWORK1: A PC-based tool for assessment of performance in a simulated work environment. Behavior Research Methods, 26(4), 421-426.

Fiedler, K. (1988). Emotional mood, cognitive style, and behavior regulation. Affect, Cognition, and Social Behavior, 100-119.

Finucane, A. M. (2011). The effect of fear and anger on selective attention. Emotion, 11(4), 970974.

Fiske, S. T., \& Taylor, S. E. (1991). Social cognition. New York: McGraw-Hill.

Forgas, J. P. (2007). When sad is better than happy: Negative affect can improve the quality and effectiveness of persuasive messages and social influence strategies. Journal of Experimental Social Psychology, 43(4), 513-528.

Fredrickson, B. L. (2003). The value of positive emotions. American scientist,91(4), 330-335. 
Fredrickson, B.L., \& Branigan, C. (2005). Positive emotions broaden the scope of attention and thought-action repertoires. Cognition and Emotion, 19, 313-332.

Gasper, K. (2004). Do you see what I see? Affect and visual information processing. Cognition and Emotion, 18(3), 405-421.

Gasper, K., \& Clore, G. L. (2002). Attending to the big picture: Mood and global versus local processing of visual information. Psychological Science, 13(1), 34-40.

Gerrards-Hesse, A., Spies, K., \& Hesse, F. W. (1994). Experimental inductions of emotional states and their effectiveness: A review. British journal of psychology, 85(1), 55-78.

Hajcak, G., McDonald, N., \& Simons, R. F. (2004). Error-related psychophysiology and negative affect. Brain and cognition, 56(2), 189-197.

Huntsinger, J. R., Clore, G. L., \& Bar-Anan, Y. (2010). Mood and global-local focus: Priming a local focus reverses the link between mood and global-local processing. Emotion, 10(5), $722-726$.

Isen, A. M. (2000). Positive affect and decision making. Handbook of emotions, 2, 417-435.

Isen, A. M., Daubman, K. A., \& Nowicki, G. P. (1987). Positive affect facilitates creative problem solving. Journal of personality and social psychology, 52(6), 1122-1131.

Isen, A. M., \& Means, B. (1983). The influence of positive affect on decision-making strategy. Social cognition, 2(1), 18-31.

Isen, A. M., \& Reeve, J. (2005). The influence of positive affect on intrinsic and extrinsic motivation: Facilitating enjoyment of play, responsible work behavior, and self-control. Motivation and Emotion, 29(4), 295-323.

Jefferies, L. N., Smilek, D., Eich, E., \& Enns, J. T. (2008). Emotional valence and arousal interact in attentional control. Psychological Science, 19(3), 290-295. 
Loewenstein, G., \& Lerner, J. S. (2003). The role of affect in decision making. In R. Davidson, K. Scherer, \& H. Goldsmith (Eds.), Handbook of affective science (pp. 619-642). New York: Oxford University Press.

Lucas, R. E., \& Diener, E. (2003). The happy worker: Hypotheses about the role of positive affect in worker productivity. In M. Burrick \& A. M. Ryan (Eds.), Personality and Work. San Francisco: Jossey-Bass.

Luu, P., Collins, P., \& Tucker, D. M. (2000). Mood, personality, and self-monitoring: negative affect and emotionality in relation to frontal lobe mechanisms of error monitoring. Journal of Experimental Psychology: General, 129(1), 43-60.

Lyubomirsky, S., King, L., \& Diener, E. (2005). The benefits of frequent positive affect: Does happiness lead to success? Psychological Bulletin, 131(6), 803-855.

Martin, M. (1990). On the induction of mood. Clinical Psychology Review, 10(6), 669-697.

Marvel, C. L., \& Paradiso, S. (2004). Cognitive and neurological impairment in mood disorders. The Psychiatric clinics of North America, 27(1), 19-36.

Mason, W., \& Suri, S. (2012). Conducting behavioral research on Amazon's Mechanical Turk. Behavior Research Methods, 44(1), 1-23.

Mayr, U., Kuhns, D., \& Rieter, M. (2013). Eye movements reveal dynamics of task control. Journal of Experimental Psychology: General, 142(2), 489-509.

Monsell, S. (2003). Task switching. Trends in Cognitive Sciences, 7, 134-140.

Morgan, B., D’Mello, S. K., Abbott, R. G., Radvansky, G. A., Haass, M., \& Tamplin, A. (2013b). Individual differences in multitasking ability and adaptability. Human Factors, 55, 776-788. doi: 10.1177/0018720812470842 
Navon, D. (1977). The forest before trees: The precedence of global features in visual perception. Cognitive Psychology, 9(3), 353-383.

Olivers, C. N., \& Nieuwenhuis, S. (2005). The beneficial effect of concurrent task-irrelevant mental activity on temporal attention. Psychological Science, 16(4), 265-269.

Olivers, C. N., \& Nieuwenhuis, S. (2006). The beneficial effects of additional task load, positive affect, and instruction on the attentional blink. Journal of Experimental Psychology: Human Perception and Performance, 32(2), 364-379.

Paolacci, G., Chandler, J., \& Ipeirotis, P. G. (2010). Running experiments on Amazon Mechanical Turk. Judgment and Decision Making, 5(5), 411-419.

Pea, R., Nass, C., Meheula, L., Rance, M., Kumar, A., Bamford, H., . . Zhou, M. (2012). Media use, face-to-face communication, media multitasking, and social well-being among 8- to 12-year-old girls. Developmental Psychology, 48, 327-336.

Posner, M. I. (1990). Hierarchical distributed networks in the neuropsychology of selective attention. In A. Carramaza (Ed.), Cognitive neuropsychology and neurolinguistics: Advances in models of cognitive function and impairment (pp. 187-210). New York: Plenum.

Proctor, R. W., Wang, D. Y., \& Pick, D. F. (1998). An empirical evaluation of the SYNWORK1 multiple-task work environment. Behavior Research Methods, Instruments, \& Computers, 30(2), 287-305.

Rand, D. G. (2012). The promise of Mechanical Turk: how online labor markets can help theorists run behavioral experiments. Journal of Theoretical Biology, 299, 172-179. doi:10.1016/j.jtbi.2011.03.004. 
Rokke, P. D., Arnell, K. M., Koch, M. D., \& Andrews, J. T. (2002). Dual-task attention deficits in dysphoric mood. Journal of abnormal psychology, 111(2), 370-379.

Rowe, G., Hirsh, J. B., \& Anderson, A. K. (2007). Positive affect increases the breadth of attentional selection. Proceedings of the National Academy of Sciences, 104(1), 383-388.

Rubinstein, J. S., Meyer, D. E., \& Evans, J. E. (2001). Executive control of cognitive processes in task switching. Journal of Experimental Psychology: Human Perception and Performance, 27(4), 763-797.

Russell, J. A., Weiss, A., \& Mendelsohn, G. A. (1989). Affect Grid: A single-item scale of pleasure and arousal. Journal of Personality and Social Psychology, 57(3), 493-502.

Sauer, J., Wastell, D. G., \& Hockey, G. R. J. (1999). Multiple-task performance on a computersimulated life support system during a space mission simulation. Acta astronautica, $44(1), 43-52$

Schaefer, A., Nils, F., Sanchez, X., \& Philippot, P. (2010). Assessing the effectiveness of a large database of emotion-eliciting films: A new tool for emotion researchers. Cognition and Emotion, 24(7), 1153-1172.

Schmitz, T. W., De Rosa, E., \& Anderson, A. K. (2009). Opposing influences of affective state valence on visual cortical encoding. The Journal of Neuroscience, 29(22), 7199-7207.

Schwarz, N. (2000). Emotion, cognition, and decision making. Cognition \& Emotion, 14(4), 433440.

Sinclair, R. C. (1988). Mood, categorization breadth, and performance appraisal: The effects of order of information acquisition and affective state on halo, accuracy, information retrieval, and evaluations. Organizational Behavior and Human Decision Processes, $42(1), 22-46$. 
Smallwood, J., Fitzgerald, A., Miles, L. K., \& Phillips, L. H. (2009). Shifting moods, wandering minds: negative moods lead the mind to wander. Emotion, 9(2), 271-276.

Solomon, R. L., \& Corbit, J. D. (1974). An opponent-process theory of motivation: I. Temporal dynamics of affect. Psychological Review, 81(2), 119-145.

Storbeck, J., \& Clore, G. L. (2007). On the interdependence of cognition and emotion. Cognition and Emotion, 21(6), 1212-1237.

Uddenberg, S., \& Shim, W. M. (2015). Seeing the world through target-tinted glasses: Positive mood broadens perceptual tuning. Emotion, 15(3), 319-328.

Van Dillen, L. F., \& Koole, S. L. (2007). Clearing the mind: a working memory model of distraction from negative mood. Emotion, 7(4), 715-723.

Van Steenbergen, H., Band, G. P., \& Hommel, B. (2010). In the mood for adaptation how affect regulates conflict-driven control. Psychological Science, 21(11), 1629-1634.

Van Wouwe, N. C., Band, G. P., \& Ridderinkhof, K. R. (2011). Positive affect modulates flexibility and evaluative control. Journal of Cognitive Neuroscience, 23(3), 524-539.

Vinski, M. T., \& Watter, S. (2013). Being a grump only makes things worse: a transactional account of acute stress on mind wandering. Frontiers in Psychology, 4, 730.

Wadlinger, H. A., \& Isaacowitz, D. M. (2006). Positive mood broadens visual attention to positive stimuli. Motivation and Emotion, 30(1), 87-99.

Wetherell, M. A., \& Sidgreaves, M. C. (2005). Secretory immunoglobulin-A reactivity following increases in workload intensity using the Defined Intensity Stressor Simulation (DISS). Stress and Health, 21(2), 99-106. 
Yang, H., \& Yang, S. (2014). Positive affect facilitates task switching in the dimensional change card sort task: Implications for the shifting aspect of executive function. Cognition and Emotion, 28(7), 1242-1254.

Zajonc, R. B. (1980). Feeling and thinking: Preferences need no inferences. American Psychologist, 35(2), 151-175.

Zajonc, R. B. (2000). Feeling and thinking: Closing the debate over the independence of affect. In J. P. Forgas (Ed.), Feeling and thinking: The role of affect in social cognition. Studies in emotion and social interaction (Vol. 2., pp. 31-58). New York: Cambridge University Press. 